\section{Tendência temporal de indicadores da prática de atividade físíca e comportamento sedentário nas capitais da Região Norte do Brasil: 2006-2013}

\author{
Time trends of physical activity and \\ sedentary behaviour in state capital of \\ North region of Brazil: 2006-2013
}

Grégore I Mielke* 1.

Danylo José Simões Costa

Sheila Rizzato Stopa 2,4

Maryane Oliveira Campos ${ }^{2}$

Demilto Yamaguchi da Pureza ${ }^{3}$

Marta Maria Alves da Silva ${ }^{2}$

\section{RESUMO}

O objetivo deste estudo foi descrever as mudanças na prática de atividade física nas capitais da região Norte entre 2006 e 2013. Foram analisados os dados de adultos (18+ anos) que fizeram parte do Sistema de Vigilância de Fatores de Risco e Proteção para Doenças Crônicas por Inquérito Telefônico (VIGITEL), das capitais de estados da região Norte do Brasil (Acre, Amazonas, Amapá, Pará, Rondônia, Roraima, Tocantins) no período de 2006 a 2013. Foram analisadas as tendências de atividade física no tempo livre, deslocamento a pé ou de bicicleta para ir/voltar do trabalho/escola, prevalência de inatividade física considerando todos os domínios (lazer, deslocamento, trabalho e tarefas domésticas), além da proporção de adultos que relataram três ou mais horas assistindo televisão por dia. Para os indicadores de atividade física no tempo livre, prevalência de adultos que assistiram televisão por três ou mais horas/dia e prevalência de inatividade física não foram observadas mudanças no período analisado. Houve uma redução na proporção de adultos que relataram se deslocar a pé ou de bicicleta para o trabalho/escola de $19,1 \%$ em 2009 para $11,8 \%$ em

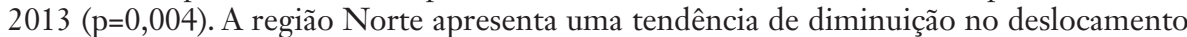
ativo semelhante à tendência nacional, enquanto que a prática de atividade física no lazer permaneceu estável. Monitorar os indicadores de atividade física no contexto regional pode auxiliar gestores no direcionamento de políticas de promoção da atividade física.

\section{PALAVRAS-CHAVE}

Estilo de vida sedentário; Esportes; Adulto; Vigilância em saúde pública; Atividade motora.

\begin{abstract}
The purpose of this study was to describe time trends in physical activity and television viewing time among adult $(18+$ years) residents in state capitals from the Brazilian Northern region. Data from 2006 to 2013 from Surveillance System Risk factors for chronic diseases and protection were analyzed by telephone survey (VIGITEL), limiting the analysis to the Northern state capitals (Acre, Amazonas, Amapá, Pará, Rondônia, Roraima, Tocantins). Trends of physical activity at leisure time, walking or biking to go/come back work/school, prevalence of physical inactivity considering all domains (leisure, travel, work and household activities), and the prevalence of adults reporting three or more hours per day watching television were described. Leisure-time physical activity, prevalence of adults watching television for three or more hours/day and prevalence of physical inactivity did not change during the studied period. There was a decrease in the proportion of adults using active commuting to go to/from work/school from $19.1 \%$ in 2009 to $11.8 \%$ in $2013(p=0.004)$. The northern region shows a decreasing trend similar to the national trends in active commuting, while leisure time physical activity remains stable. Surveillance of physical activity in the regional context could assist policy makers in targeting policies to promote physical activity.
\end{abstract}

\section{KEYWORDS}

Sedentary Lifestyle; Sports; Adult; Public Health Surveillance; Motor Activity.
Rev Bras Ativ Fís Saúde p. 130-140 DOI

http://dx.doi.org/10.12820/rbafs.v.20n2p130

1 Programa de Pós-Graduação em Epidemiologia Universidade Federal de Pelotas. Pelotas, RS, Brasil

2 Departamento de Doenças e Agravos Não Transmissíveis, e Promoção da Saúde, Secretaria de Vigilância em Saúde do Ministério da Saúde. Brasília, DF, Brasil

3 Programa de Pós Graduação em Ciências da Saúde, Universidade Federal do Amapá. Macapá, AP, Brasil

4 Departamento de Epidemiologia. Faculdade de Saúde Pública da Universidade de São Paulo FSP-USP. São Paulo-SP, Brasil. 


\section{INTRODUCCÃO}

Nos últimos anos o Brasil tem avançado bastante nas pesquisas na área da atividade física e saúde ${ }^{1,2}$. Hoje, podemos encontrar dados sobre prevalências de prática de atividade física em uma gama de cidades brasileiras. Apesar deste panorama, algumas lacunas permanecem: a) a comparação entre as prevalências de atividade física em diferentes regiões nem sempre é possível devido aos diferentes instrumentos utilizados na mensuração; b) a distribuição dos estudos sobre prática de atividade física não ocorre aleatoriamente no Brasil, existindo uma concentração de informações nas regiões sul e sudeste; c) até o momento, poucos estudos têm investigado as tendências temporais de atividade física no Brasil ${ }^{3}$.

Em uma recente revisão sobre a evolução da pesquisa epidemiológica em atividade física no Brasil, Ramires e colegas mostraram que dentre todos os artigos sobre atividade física no Brasil publicado entre 2006 e 2013, apenas dois eram com dados da região Norte do Brasil ${ }^{3}$. Com a implementação do Sistema de Vigilância de Fatores de Risco e Proteção para Doenças Crônicas Não Transmissíveis por Inquérito Telefônico, (VIGITEL), o Brasil dispõe de dados para todo território nacional e torna-se um dos poucos países de renda média e baixa a terem dados nacionais sobre tendências de atividade física. Por exemplo, considerando as 26 capitais e o Distrito Federal, a proporção de adultos que relataram praticar atividade física no lazer por pelo menos $30 \mathrm{mi}$ nutos por dia em cinco ou mais dias na semana aumentou de 12,8\% em 2006 para $14,9 \%$ em $2012{ }^{4}$. Também foi evidenciada uma redução do deslocamento ativo para o trabalho e na proporção de adultos que assistem televisão por mais de três horas por dia ${ }^{4}$.

Além da existência de dados nacionais oriundos do VIGITEL ${ }^{4,5}$, também é possível que seja apresentada uma visão detalhada das tendências temporais de atividade física de acordo com regiões do Brasil. Devido às diferenças demográficas, no estado de saúde e nos modos de vida existentes entre as cinco grandes regiões do Brasil, compreender como os indicadores de atividade física e comportamento sedentário estão mudando em cada região do Brasil torna-se importante, uma vez que a prática de atividade física é bastante influenciada por questões socioculturais ${ }^{6}$, as quais apresentam as peculiaridades regionais. Assim, através do conhecimento das tendências temporais de atividade física de acordo com as regiões do Brasil, o planejamento de políticas de promoção da prática de atividade física pode ser maximizado.

Tendo como objetivo desta edição especial à descrição das tendências de prática de atividade física de acordo com regiões do Brasil, o objetivo deste estudo foi descrever as mudanças nos indicadores da prática de atividade física e do comportamento sedentário nas capitais da região Norte entre 2006 e 2013.

\section{MÉTODOS}

Este estudo utilizou dados das capitais da região Norte coletados pelo VIGITEL entre os anos de 2006 e 2013. Resumidamente, por ano em cada uma das sete capitais dos estados que compõem a região Norte do Brasil (Acre, Amazonas, Amapá, Pará, Rondônia, Roraima, Tocantins) foram entrevistados 2.000 adultos (18+ anos). Foram realizadas entrevistas telefônicas e coletadas 
informações sobre fatores de risco e proteção para doenças crônicas, dentre estes, indicadores sobre a prática de atividade física e tempo assistindo televisão. Detalhes adicionais sobre o processo de amostragem e questões operacionais estão disponíveis no website da Secretaria de Vigilância em Saúde do Ministério da Saúde e em outras publicações ${ }^{7}$.

Desde que foi criado em 2006, o VIGITEL tem coletado informações sobre a prática de atividade física em todos os domínios. Além do mais, o tempo diário assistindo televisão é mensurado como uma estimativa de comportamento sedentário. Para este estudo, foram considerados os seguintes indicadores:

- Ativo no tempo livre: respondentes que praticaram pelo menos 150 minutos semanais de atividade física de intensidade leve ou moderada ou pelo menos 75 minutos semanais de atividade física de intensidade vigorosa ( $\operatorname{sim} /$ não) ${ }^{8}$, 9. Este indicador é estimado a partir das questões: "Nos últimos três meses, o(a) Sr(a) praticou algum tipo de exercício físico ou esporte?", "Qual o tipo principal de exercício físico ou esporte que o(a) $\operatorname{Sr}($ a) praticou?", "O(a) Sr(a) pratica o exercício pelo menos uma vez por semana?", "Quantos dias por semana o(a) Sr(a) costuma praticar exercício físico ou esporte?" e "No dia que o(a) Sr(a) pratica exercício ou esporte, quanto tempo dura esta atividade?". Devido a modificações nas opções de resposta a partir do inquérito em 2009, iremos considerar o período de análise entre 2009 a 2013. Para a construção deste indicador foram consideradas atividades de intensidade leve/moderada a caminhada, caminhada em esteira, musculação, hidroginástica, ginástica em geral, natação, artes marciais, ciclismo e voleibol; corrida, corrida em esteira, ginástica aeróbica, futebol, basquetebol e tênis foram classificados como práticas de intensidade vigorosa.

- Ativos no deslocamento: adultos que se deslocaram para o trabalho ou escola/faculdade de bicicleta ou caminhando e que despendem pelo menos 30 minutos diários no percurso de ida e volta (sim/não). Para este indicador foram consideradas as questões sobre deslocamento para trabalho e/ou curso e/ou escola a seguir: "Para ir ou voltar ao seu trabalbo, faz algum trajeto a pé ou de bicicleta?", "Quanto tempo o(a) Sr(a) gasta para ir e voltar neste trajeto (a pé ou de bicicleta)?", "Atualmente, o(a) Sr(a) está frequentando algum curso/ escola ou leva alguém em algum curso/escola?" " "Para ir ou voltar a este curso ou escola, faz algum trajeto a pé ou de bicicleta?".

- Fisicamente inativos: Foram considerados fisicamente inativos aqueles participantes que não realizaram esforços físicos intensos no trabalho, relataram não serem responsáveis pela limpeza pesada de casa, que não tenham se deslocado para o trabalho ou curso/escola caminhando ou de bicicleta perfazendo um mínimo de 10 minutos por trajeto por dia e que não praticaram qualquer atividade física no tempo livre nos últimos três meses ( $\operatorname{sim} /$ não). Este indicador foi construído com base na combinação de respostas dadas para as questões: "Nos últimos três meses, o(a) Sr(a) praticou algum tipo de exercício físico ou esporte?"; "No seu trabalho, o(a) Sr(a) anda bastante a pe??", ou "No seu trabalho, o(a) Sr (a) carrega peso ou faz outra atividade pesada?"; "O (a) Sr(a) costuma ir a pé ou de bicicleta de casa para o trabalho?" e "Quanto tempo o(a) Sr(a) gasta para ir e voltar do trabalho (a pé ou de bicicleta)?”; "Atualmente, o(a) Sr(a) está frequentando algum curso/escola ou leva alguém em algum curso/escola?" e "Para ir ou voltar a este curso ou escola, faz algum trajeto a pé ou de bicicleta?"; e "Quem costuma fazer a faxina da sua casa?" ou "Quem fica com a parte mais pesada da faxina, quando tem ajuda?". 
- Tempo de televisão: Proporção de participantes que relataram assistir três ou mais horas de televisão por dia. O indicador foi construído com base na resposta dada para a questão "Em média, quantas horas por dia o(a) $\operatorname{Sr}($ a) costuma ficar assistindo televisão?".

As análises de tendência para cada um dos indicadores foram realizadas de acordo com sexo (masculino; feminino), grupos de idade (18-24; 25-34; 35-44; 45-54; 55-64; 65+ anos), escolaridade em anos completos (0-8; 9-11; 12+) e capitais (Belém, Boa Vista, Macapá, Manaus, Palmas, Porto Velho e Rio Branco). Em razão das modificações no questionário a partir do inquérito de 2009, as análises para o indicador de atividade física no tempo livre e no deslocamento serão apresentadas apenas para o período entre 2009 e 2013. Foram utilizados modelos de regressão linear, tendo cada indicador de atividade física como variável dependente e o ano do levantamento como variável independente. Foram consideradas mudanças significativas, aquelas cujo coeficiente de regressão para a variável ano do inquérito apresentou um valor $\mathrm{p}<0,05$. Todas as estimativas provenientes do VIGITEL são calculadas com a utilização de pesos pós-estratificação, objetivando igualar a composição sociodemográfica estimada para a população de adultos de cada capital. Estes pesos são compostos pelas variáveis: sexo, faixa etária e escolaridade; calculados pelo método "rake" ${ }^{10}$. Todas as análises foram realizadas no pacote estatístico Stata, versão 12.1 (Stata Corp., College Station, EUA). Este estudo foi aprovado pela Comissão Nacional de Ética em Pesquisa em Seres Humanos (CONEP) 13081/2008 e 355590/2013.

\section{RESULTADOS}

Fizeram parte das análises 104.168 participantes entrevistados entre 2006 e 2013, variando de 14.163 em 2010 a 7.858 entrevistados em 2013. A menor taxa de resposta do VIGITEL na região Norte foi de 57,8\% em Belém em 2011, sendo a maior taxa de reposta de 87,7\% na cidade de Palmas em 2009. A Figura 1 descreve as tendências temporais para cada um dos indicadores analisados. Observamos uma redução na proporção de adultos que relataram se deslocar a pé ou de bicicleta para o trabalho/escola de 19,1\% em 2009 para 11,8\% em 2013 ( $\mathrm{p}=0,004$ ). A proporção de ativos no tempo livre, fisicamente inativos e que assistem televisão por três horas ou mais por dia permaneceu estável no período analisado.

A análise de tendência temporal de atividade física no tempo livre de acordo com variáveis sociodemográficas não apresentou diferenças, quando comparado às tendências da região. De uma forma geral, a prevalência de ativos no tempo livre foi maior em homens, mais jovens e aqueles com maior escolaridade, quando comparado aos seus pares. (Tabela 1). Considerando apenas o ano de 2013, a prevalência de ativos no tempo livre variou de 40,2\% em Boa Vista e Palmas a $31,6 \%$ em Porto Velho. Houve uma tendência significativa de aumento na proporção de ativos no tempo livre em Palmas (32,6\% em 2009 para 40,2\% em 2013) e Rio Branco (27,6\% em 2009 para 36,2\% em 2013). (Tabela 1).

A Tabela 2 mostra as tendências temporais de ativos no deslocamento de acordo com sexo, grupos de idade, escolaridade e para cada uma das capitais da região Norte. Observa-se uma tendência significativa de redução na proporção de ativos no deslocamento em todos os subgrupos populacionais. Esta 
tendência foi mais acentuada entre aqueles participantes com menor escolaridade. Em média, entre aqueles com 0-8 anos de escolaridade a diminuição anual foi de 2,7 pontos percentuais $(\mathrm{p}=0,008)$, enquanto que entre aqueles com mais de 12 anos de estudo, o declínio médio foi de 0,8 pontos percentuais por ano $(\mathrm{p}=0,047)$. Em 2013 Palmas foi a capital que apresentou a menor proporção de ativos no deslocamento $(5,1 \%)$ enquanto que Belém foi a capital com maior proporção de ativos no deslocamento $(13,7 \%)$.

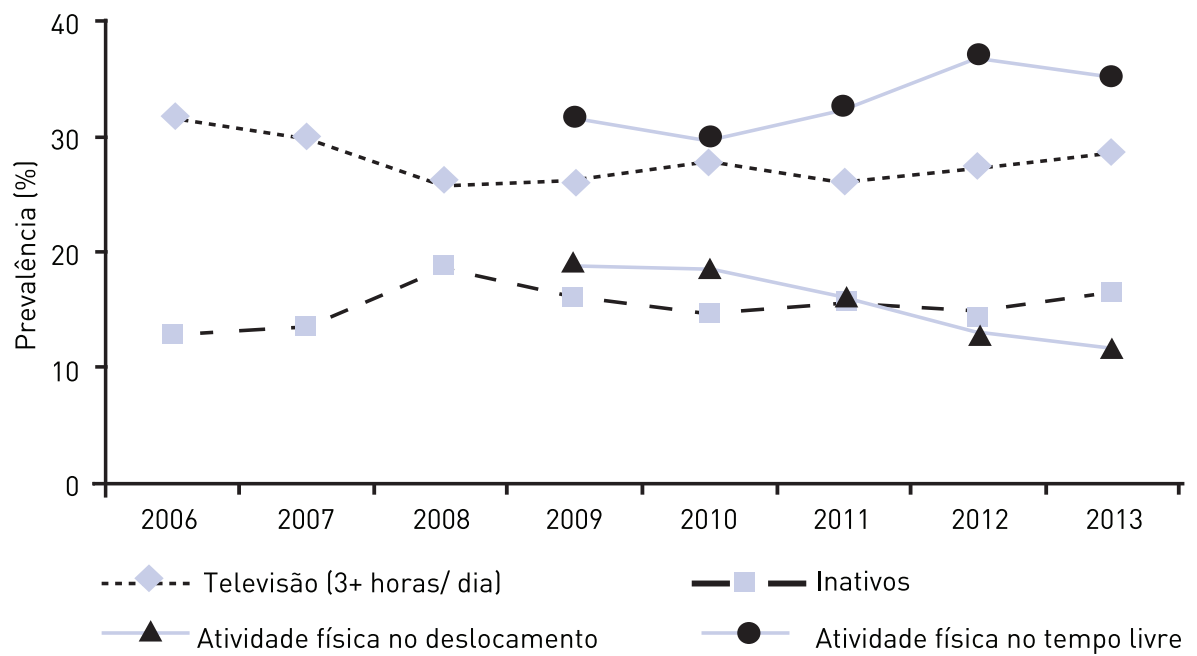

FIGURA 1 - Tendências temporais de atividade física e tempo de televisão na região Norte do Brasil. VIGITEL: 2006-2013.

TABELA 1 - Tendência temporal de atividade física no tempo livre de acordo com sexo, idade, escolaridade e capitais da região Norte do Brasil. VIGITEL: 2006-2013

\begin{tabular}{lccccccc}
\hline $\begin{array}{l}\text { Variáveis } \\
\text { Sexo }\end{array}$ & 2009 & 2010 & 2011 & 2012 & 2013 & Mudança (p.p) & Valor $p$ \\
\hline Masculino & 41,5 & 39,8 & 42,7 & 45,8 & 43,4 & $+1,0$ & 0,194 \\
\hline Feminino & 22,5 & 20,8 & 23,8 & 29,4 & 27,5 & $+1,9$ & 0,085 \\
\hline Idade & & & & & & & \\
\hline 18-24 & 44,3 & 40,3 & 46,6 & 48,9 & 51,5 & $+2,3$ & 0,071 \\
\hline $25-34$ & 34,2 & 35,3 & 35,7 & 41,6 & 38,3 & $+1,5$ & 0,126 \\
\hline $35-44$ & 28,6 & 24,7 & 29,5 & 35,7 & 31,0 & $+1,6$ & 0,259 \\
\hline $45-54$ & 24,8 & 21,3 & 26,5 & 29,6 & 28,9 & $+1,7$ & 0,121 \\
\hline $55-64$ & 19,1 & 23,1 & 20,9 & 26,9 & 22,8 & $+1,1$ & 0,276 \\
\hline 65+ & 18,8 & 17,2 & 17,4 & 19,1 & 17,5 & $-0,1$ & 0,840 \\
\hline Escolaridade & & & & & & & \\
\hline 0-8 & 22,0 & 21,0 & 23,8 & 28,3 & 21,8 & $+0,7$ & 0,538 \\
\hline 9-11 & 37,8 & 35,4 & 38,2 & 39,4 & 40,4 & $+0,9$ & 0,128 \\
\hline 12+ & 42,3 & 37,9 & 41,3 & 48,7 & 47,1 & $+2,0$ & 0,160 \\
\hline Cidades & & & & & & & \\
\hline Belém & 33,2 & 32,4 & 34,8 & 38,3 & 36,2 & $+1,2$ & 0,107 \\
\hline Boa Vista & 31,6 & 28,2 & 34,0 & 35,9 & 40,2 & $+2,5$ & 0,054 \\
\hline Macapá & 31,6 & 33,6 & 30,4 & 37,5 & 36,2 & $+1,3$ & 0,196 \\
\hline Manaus & 31,0 & 28,3 & 32,3 & 37,8 & 33,1 & $+1,4$ & 0,262 \\
\hline Palmas & 32,6 & 33,0 & 38,1 & 38,1 & 40,2 & $+2,0$ & 0,016 \\
\hline Porto Velho & 30,4 & 27,3 & 28,4 & 34,5 & 31,6 & $+1,0$ & 0,350 \\
\hline Rio Branco & 27,6 & 25,7 & 31,0 & 32,6 & 36,2 & $+2,4$ & 0,027 \\
\hline & & & & & & & \\
\hline
\end{tabular}


TABELA 2 - Tendência temporal de atividade física no deslocamento de acordo com sexo, idade, escolaridade e capitais da região Norte do Brasil. VIGITEL: 2006-2013

\begin{tabular}{lccccccc}
\hline Variáveis & 2009 & 2010 & 2011 & 2012 & 2013 & Mudança (p.p) & Valor $p$ \\
\hline Sexo & & & & & & & \\
\hline Masculino & 20,2 & 20,0 & 17,6 & 15,5 & 12,9 & $-1,9$ & 0,005 \\
\hline Feminino & 18,1 & 17,8 & 15,3 & 11,5 & 10,9 & $-2,1$ & 0,009 \\
\hline Idade & & & & & & & \\
\hline $18-24$ & 19,4 & 18,4 & 18,9 & 13,8 & 14,1 & $-1,5$ & 0,050 \\
\hline $25-34$ & 20,0 & 20,2 & 19,2 & 13,4 & 11,9 & $-2,3$ & 0,029 \\
\hline $35-44$ & 20,4 & 20,5 & 15,1 & 15,6 & 12,7 & $-2,0$ & 0,023 \\
\hline $45-54$ & 22,5 & 22,4 & 17,9 & 15,4 & 13,4 & $-2,5$ & 0,005 \\
\hline $55-64$ & 15,9 & 16,4 & 10,0 & 11,1 & 8,1 & $-2,1$ & 0,038 \\
\hline 65+ & 6,8 & 4,0 & 5,8 & 3,8 & 4,1 & $-0,6$ & 0,220 \\
\hline Escolaridade & & & & & & & \\
\hline 0-8 & 21,8 & 22,1 & 18,7 & 14,8 & 12,2 & $-2,7$ & 0,008 \\
\hline 9-11 & 19,2 & 18,7 & 17,4 & 14,1 & 13,0 & $-1,7$ & 0,008 \\
\hline $12+$ & 11,7 & 11,4 & 9,0 & 9,4 & 8,9 & $-0,8$ & 0,047 \\
\hline Cidades & & & & & & & \\
\hline Belém & 20,3 & 21,5 & 17,8 & 16,5 & 13,7 & $-1,8$ & 0,022 \\
\hline Boa Vista & 16,5 & 14,6 & 11,7 & 14,3 & 9,1 & $-1,5$ & 0,082 \\
\hline Macapá & 21,1 & 21,2 & 18,8 & 15,0 & 12,6 & $-2,3$ & 0,009 \\
\hline Manaus & 18,2 & 17,6 & 16,2 & 10,7 & 11,5 & $-2,0$ & 0,029 \\
\hline Palmas & 14,3 & 13,5 & 10,1 & 10,5 & 5,1 & $-2,1$ & 0,020 \\
\hline Porto Velho & 18,8 & 18,6 & 16,7 & 14,0 & 11,7 & $-1,9$ & 0,006 \\
\hline Rio Branco & 21,6 & 18,7 & 16,1 & 13,1 & 12,0 & $-2,5$ & 0,001 \\
\hline
\end{tabular}

TABELA 3 - Tendência temporal de fisicamente inativos de acordo com sexo, idade, escolaridade e capitais da região Norte do Brasil, VIGITEL: 2006-2013

\begin{tabular}{|c|c|c|c|c|c|c|c|c|c|c|}
\hline Variáveis & 2006 & 2007 & 2008 & 2009 & 2010 & 2011 & 2012 & 2013 & $\begin{array}{c}\text { Mudança } \\
(p, p)\end{array}$ & Valor $p$ \\
\hline \multicolumn{11}{|l|}{ Sexo } \\
\hline Masculino & 13,8 & 15,0 & 17,1 & 15,3 & 12,9 & 14,8 & 13,3 & 15,8 & $-0,1$ & 0,851 \\
\hline Feminino & 12,0 & 12,4 & 20,6 & 17,2 & 16,4 & 17,0 & 15,6 & 17,3 & $+0,5$ & 0,283 \\
\hline \multicolumn{11}{|l|}{ Idade } \\
\hline $18-24$ & 10,0 & 12,8 & 17,8 & 14,3 & 14,9 & 12,8 & 11,7 & 14,2 & $+0,1$ & 0,779 \\
\hline $25-34$ & 9,0 & 9,9 & 14,8 & 13,0 & 10,3 & 12 & 12,3 & 12,7 & $+0,3$ & 0,311 \\
\hline $35-44$ & 10,4 & 12,5 & 14,2 & 11,9 & 10,6 & 13,3 & 11,2 & 12,7 & $+0,1$ & 0,772 \\
\hline $45-54$ & 12,8 & 11,5 & 21,7 & 15,2 & 15,1 & 15,8 & 14 & 14,4 & $+0,1$ & 0,894 \\
\hline $55-64$ & 18,2 & 19,4 & 23,0 & 23,9 & 17,9 & 23,7 & 19,4 & 26,7 & $+0,7$ & 0,203 \\
\hline $65+$ & 42,9 & 34,8 & 45,0 & 44,0 & 41,6 & 40,8 & 36,6 & 43,8 & $+0,0$ & 0,996 \\
\hline \multicolumn{11}{|l|}{ Escolaridade } \\
\hline $0-8$ & 14,3 & 15 & 20,9 & 19,5 & 16,3 & 19,2 & 18,2 & 21,5 & $+0,7$ & 0,091 \\
\hline $9-11$ & 9,1 & 11,1 & 14,8 & 12,3 & 12,3 & 12,3 & 12 & 13,2 & $+0,3$ & 0,252 \\
\hline $12+$ & 17,9 & 16,4 & 23,2 & 17,4 & 16,6 & 16,3 & 12,9 & 15,2 & $-0,7$ & 0,136 \\
\hline \multicolumn{11}{|l|}{ Cidades } \\
\hline Belém & 14,6 & 14,4 & 21,4 & 15,9 & 16,3 & 16,0 & 15,6 & 16,7 & $+0,1$ & 0,878 \\
\hline Boa Vista & 11,8 & 12,2 & 15,9 & 16,2 & 16,0 & 13,3 & 14,3 & 16,3 & $+0,4$ & 0,176 \\
\hline Macapá & 13,0 & 11,6 & 16,0 & 18,0 & 14,8 & 19,5 & 15,2 & 18,4 & $+0,8$ & 0,067 \\
\hline Manaus & 11,2 & 13,6 & 18,6 & 16,5 & 12,5 & 15,7 & 13,8 & 16 & $+0,3$ & 0,524 \\
\hline Palmas & 12,0 & 12,7 & 13,6 & 14,7 & 13,7 & 15,1 & 12,2 & 17,5 & $+0,5$ & 0,091 \\
\hline Porto Velho & 12,2 & 12,5 & 16,6 & 14,2 & 14,0 & 14,4 & 12,8 & 16,2 & $+0,3$ & 0,319 \\
\hline Rio Branco & 15,9 & 15,7 & 19,6 & 18,4 & 19,8 & 17,5 & 16,4 & 17,7 & $+0,1$ & 0,623 \\
\hline
\end{tabular}


TABELA 4 - Tendência temporal de tempo assistindo televisão (3+horas dia) de acordo com sexo, idade, escolaridade e capitais da região Norte do Brasil, VIGITEL: 2006-2013

\begin{tabular}{lccccccccccc}
\hline Variáveis & 2006 & 2007 & 2008 & 2009 & 2010 & 2011 & 2012 & 2013 & $\begin{array}{c}\text { Mudança } \\
\text { (p,p) }\end{array}$ & Valor $p$ \\
\hline Sexo & & & & & & & & & & \\
\hline Masculino & 31,1 & 29,6 & 27,1 & 26,4 & 30,2 & 28,2 & 29,1 & 29,4 & $-0,9$ & 0,749 \\
\hline Feminino & 32,5 & 30,5 & 25,4 & 25,5 & 26,4 & 24,3 & 25,8 & 28,3 & $-0,7$ & 0,259 \\
\hline Idade & & & & & & & & & & \\
\hline 18-24 & 39,7 & 39,4 & 31,7 & 33,3 & 32,3 & 31,2 & 30,9 & 32,3 & $-1,2$ & 0,021 \\
\hline 25-34 & 31,0 & 29,7 & 26,9 & 25,2 & 29,8 & 26,6 & 26,8 & 30,1 & $-0,2$ & 0,575 \\
\hline 35-44 & 28,6 & 26,8 & 21,3 & 23,9 & 26,3 & 25,0 & 28,8 & 27,8 & 0,2 & 0,629 \\
\hline 45-54 & 26,2 & 26,0 & 24,9 & 22,2 & 25,3 & 22,4 & 23,4 & 25,2 & $-0,3$ & 0,261 \\
\hline 55-64 & 26,9 & 22,2 & 23,1 & 22,8 & 24,9 & 23,1 & 26,3 & 27,4 & 0,3 & 0,367 \\
\hline 65+ & 35,3 & 27,6 & 27,2 & 24,4 & 24,8 & 24,5 & 25,1 & 26,8 & $-0,9$ & 0,084 \\
\hline Escolaridade & & & & & & & & & & \\
\hline 0-8 & 31,7 & 29,3 & 25,2 & 26,0 & 26,0 & 23,3 & 24,9 & 28,2 & $-0,6$ & 0,150 \\
\hline 9-11 & 33,7 & 33,4 & 28,6 & 28,4 & 32,3 & 30 & 31,6 & 32,1 & $-0,1$ & 0,683 \\
\hline 12+ & 27,4 & 22,9 & 23,1 & 19,8 & 23,9 & 24,1 & 23 & 23,4 & $-0,2$ & 0,492 \\
\hline Cidades & & & & & & & & & & \\
\hline Belém & 34,9 & 33,0 & 28,9 & 26,9 & 31,6 & 29,9 & 31,5 & 31,2 & $-0,3$ & 0,463 \\
\hline Boa Vista & 28,0 & 29,8 & 24,1 & 24,6 & 25,2 & 23,7 & 22,5 & 28,0 & $-0,4$ & 0,296 \\
\hline Macapá & 37,0 & 32,6 & 28,7 & 29,3 & 31,5 & 31,5 & 30,4 & 34,1 & $-0,3$ & 0,594 \\
\hline Manaus & 30,7 & 29,3 & 24,7 & 25,3 & 27,2 & 24,0 & 26,4 & 27,5 & $-0,4$ & 0,237 \\
\hline Palmas & 27,2 & 24,1 & 22,5 & 21,5 & 21,4 & 21,5 & 18,9 & 22,7 & $-0,7$ & 0,039 \\
\hline Porto Velho & 28,2 & 27,4 & 25,0 & 28,9 & 25,1 & 23,3 & 23,7 & 26,3 & $-0,5$ & 0,135 \\
\hline Rio Branco & 27,3 & 24,7 & 23,3 & 21,6 & 26,2 & 23,9 & 25,8 & 27,9 & $+0,2$ & 0,598 \\
\hline & & & & & & & & & & \\
\hline
\end{tabular}

A tabela 4 descreve a proporção de adultos que relataram assistir televisão por três ou mais horas por dia, de acordo com as variáveis sociodemográficas analisadas. Observamos uma tendência de redução média de 1,5 pontos percentuais ao ano $(\mathrm{p}=0,021)$ no indicador analisado apenas entre aqueles com 18-24 anos. Entre os demais subgrupos populacionais, não foram observadas mudanças. Em relação às capitais, Palmas foi a única cidade na qual houve uma tendência de diminuição na proporção de adultos que assistiram televisão por três ou mais horas diárias, com redução média anual de 0,7 pontos percentuais $(\mathrm{p}=0,039)$. Na cidade de Palmas, esta redução ocorreu apenas entre as mulheres (dados não mostrados).

\section{DISCUSSÃO}

Este estudo mostrou como os indicadores de atividade física e o tempo de televisão estão mudando ao longo do tempo nas capitais da região Norte do Brasil. Frente ao atual estado da arte da pesquisa em atividade física no Brasil, este é um dos primeiros estudos a centrar a discussão na região Norte. Em nosso estudo, observamos uma estabilidade na prática de atividade física no lazer, na inatividade física considerando todos os domínios e na proporção de adultos que relataram assistir por mais de três horas diárias. Por outro lado, houve uma tendência de diminuição da proporção de adultos que deslocaram para o trabalho ou escola de forma ativa.

$\mathrm{Na}$ análise estratificada por sexo, idade, escolaridade e por capitais da região Norte, não foram observadas tendências de mudança no indicador de fisicamente inativos. (Tabela 3) Entre 2006 e 2013, a proporção de fisicamente inativos foi pra- 
ticamente à mesma entre homens e mulheres. Em relação aos grupos de idade, em $2009,14,2 \%$ dos participantes com idade entre 18-24 anos foram considerados fisicamente inativos, enquanto que entre aqueles com 65 anos ou mais, tal proporção foi de aproximadamente $44 \%$. De uma forma geral, a prevalência de fisicamente inativos variou pouco entre as capitais da região durante o período analisado.

Os resultados devem ser compreendidos dentro de um contexto socioeconômico e cultural que fazem parte da região Norte, os quais são determinantes importantes de comportamentos, dentre eles a prática de atividade física. Nessa perspectiva, cabe destacar que a região Norte é aquela com maior área territorial e também a região com menor população dentre as cinco macrorregiões da federação ${ }^{11}$, com uma taxa de urbanização de $73,5 \%$ no conjunto de cidades e superior a $95 \%$ nas capitais. De todas as regiões é aquela que apresenta a menor proporção de pessoas com 60 anos ou mais ${ }^{11}$. Historicamente foi uma região que recebeu pouca atenção política e, de uma forma geral, apresenta piores indicadores de saúde quando comparado às outras regiões do Brasil ${ }^{12}$. Em relação ao perfil de mortalidade, diferente do Brasil onde as doenças do aparelho circulatório respondem pela maior proporção de mortalidade, na região Norte, esta é a segunda causa ${ }^{12}$.

Avaliações a nível nacional têm mostrado um aumento da proporção de adultos que praticam atividade física no lazer ${ }^{4,13}$. Analisando dados das capitais da região Sudeste, Malta e colegas mostraram um aumento na prevalência de ativos no lazer ${ }^{14}$. No nosso estudo, embora a prevalência de ativos no tempo livre tenha aumentado em termos numéricos entre 2009 e 2013 entre todos os subgrupos populacionais, tais mudanças não foram significativas. Embora não se possa estabelecer uma relação direta entre o desenvolvimento da área de pesquisa em atividade física e saúde e os níveis populacionais de atividade física na região Norte, cabe destacar que o menor desenvolvimento de pesquisas e intervenções em atividade física na região podem estar refletindo nesses indicadores ${ }^{3,15}$. Por exemplo, até 2013 não havia nenhum projeto em vigência financiado pelo Conselho Nacional de Desenvolvimento Científico e Tecnológico para o desenvolvimento da subárea de pesquisa em atividade física e saúde ${ }^{15}$. Da mesma forma, não havia nenhum programa de pós-graduação com pesquisa em atividade física e saúde na região Norte ${ }^{16}$. Em relação aos programas de atividade física financiados pelo Ministério da Saúde até 2009 , apenas $8,7 \%$ das cidades da região Norte apresentavam intervenções financiadas, sendo o menor percentual entre todas as regiões ${ }^{17}$.

A redução da proporção de adultos que se deslocam a pé ou de bicicleta para o trabalho e/ou escola, segue uma tendência nacional ${ }^{4}$. Dentre todos os indicadores de atividade física analisados, é um dos mais influenciados pelas mudanças socioeconômicas ocorridas nos últimos anos. Em nosso estudo a redução mais acentuada ocorreu entre adultos com menor escolaridade. Entre adultos com oito anos ou menos de escolaridade a prevalência de deslocamento ativo mudou de $21,8 \%$ em 2009 para $12,2 \%$ em 2013, enquanto que entre aqueles com 12 anos ou mais de escolaridade houve uma mudança de 11,7\% em 2009 para $8,9 \%$ em 2013. O aumento no poder de compra, a facilidade no acesso a veículos automotores, principalmente entre grupos com menor poder aquisitivo, aliado ao pouco investimento no planejamento urbano das cidades para implementação de ciclovias e as condições nas vias urbanas na maioria das capitais podem auxiliar a explicar estas mudanças. 
O VIGITEL tem empregado em seu monitoramento o tempo de televisão como um indicador de comportamento sedentário. No conjunto de dados analisados, não foi observada nenhuma mudança na proporção de adultos que assistem televisão por mais de três horas por dia. Obervando os dados para o conjunto de capitais brasileiras, foi obervada uma redução anual de 5\% entre 2006 e 2009 e uma estabilização entre 2010 e $2012^{4}$. É importante destacar que, embora o tempo de televisão seja frequentemente utilizado como indicador de comportamento sedentário, este é apenas um domínio do tempo total de comportamento sedentário, o qual apresenta padrões diferentes de outros comportamentos sedentários ${ }^{18}$. Entretanto, apesar das limitações, a existência de um indicador como um avaliado de forma semelhante ao longo do tempo, pode nos indicar um panorama das mudanças ocorridas nos últimos anos e estabelecer possíveis direcionamentos para a mudança de indicadores inversamente associados ao comportamento ativo da população.

A prevalência de adultos fisicamente inativos permaneceu estável entre 2006 e 2013, sendo constantemente maior entre adultos mais velhos. Um fato interessante foi a mudança na relação entre escolaridade e prevalência de inatividade física. Em 2006, a prevalência era similar entre adultos mais e menos escolarizados, porém em 2013 observamos uma diferença considerável no indicador, sendo que $21 \%$ dos adultos com oito anos ou menos de escolaridade foram classificados como fisicamente inativos, enquanto essa proporção foi de $15,2 \%$ entre aqueles mais escolarizados. Semelhante ao indicador de atividade física no deslocamento, este indicador é bastante influenciado por mudanças socioeconômicas e na forma de organização do trabalho. Dessa forma, com a redução da necessidade de trabalhos que envolvam demanda física pesada, principalmente entre adultos com menor escolaridade, era esperado essa mudança de perfil. Um padrão de mudança na prevalência de inatividade física em todos os domínios de acordo com grau de escolaridade semelhante foi encontrado em Pelotas-RS ${ }^{19}$.

Algumas limitações devem ser pontuadas neste estudo. A cobertura de linhas telefônicas fixas nas capitais da região Norte está entre as mais baixas do país e com o advento da massificação da utilização de aparelhos celulares, a adesão a serviços telefônicos residenciais diminui ao passar dos anos. Outra limitação do estudo diz respeito a extrapolação dos resultados para o conjunto da cidades da região norte. Aproximadamente apenas $31 \%$ da população total da região residem nas capitais. Considerando este dado e a taxa de cobertura de telefone fixo, as nossas estimativas representam apenas $9 \%$ da população da região norte. Para tentar minimizar a baixa cobertura, foram utilizados procedimentos estatísticos que visam extrapolar os dados para a população não coberta por linhas telefônicas fixas. Apesar destas limitações, acreditamos que os resultados apresentados fornecem uma estimativa razoável do cenário de indicadores de atividade física da região norte, principalmente se consideramos o baixo número de informações sobre prática de atividade física disponíveis na região.

Em resumo, os dados da região Norte mostraram uma estabilidade nos indicadores de atividade física, com exceção do deslocamento ativo, o qual apresentou um declínio no período analisado. Além do mais, foram ratificadas desigualdades nos indicadores de atividade física e tempo de televisão de acordo com sexo, idade e escolaridade. Frente a este cenário, é necessária uma discussão aprofundada acerca da promoção da atividade física no Brasil, con- 
siderando as especificidades de cada região. Nesse sentido, o Plano de Ações Estratégicas para o Enfrentamento de Doenças Crônicas Não Transmissíveis

${ }^{20}$ e a Política Nacional de Promoção da Saúde ${ }^{21}$ tem colocado a promoção da atividade física como uma das prioridades no cenário nacional. Dessa forma, políticas de restruturação de sistemas de transporte visando o deslocamento ativo, além do fortalecimento de políticas capazes de promover a prática de atividade física de forma agradável, segura e que apresente sentido para o indivíduo são necessárias.

\section{REFERÊNCIAS}

1. Kienteka M, Stocchero CMA, Fueyo LF, Andrade LMA, Peixoto MB. Atividade física e saúde: produção científica em periódicos da Educação Física no Brasil. Rev Bras de Ativ Fís Saúde. 2014;19(4):410-6.

2. Hallal PC, Dumith SdC, Bastos JP, Reichert FF, Siqueira FV, Azevedo MR. Evoluçãoda pesquisa epidemiológica em atividade física no Brasil: revisão sistemática. Rev de Saúde Pública. 2007;41:453-60.

3. Ramires VV, Becker LA, Sadovsky ADI, Zago AM, Bielemann RM, Gerra PH. Evolução da pesquisa epidemiológica em atividade física e comportamento sedentário no Brasil: atualização de uma revisão sistemática. Rev Bras de Ativ Fís Saúde. 2014;19(5):529-47.

4. Mielke GI, Hallal PC, Malta DC, Lee, I-Min. Time trends of physical activity and television viewing time in Brazil: 2006-2012. Int J Behav Nutr Phys Act. 2014; 15; 11:101.

5. Hallal PC, Knuth AG, Reis RS, Rombaldi AJ, Malta DC, Iser BPM et al . Tendências temporais de atividade física no Brasil (2006-2009). Rev Bras Epidemiol. 2011; 14(Supl 1): 53-60.

6. Bauman AE, Reis RS, Sallis JF, Wells JC, Loos RJ, Martin BW; Lancet Physical Activity Series Working Group. Correlates of physical activity: why are some people physically active and others not? Lancet. 2012; 380(9838):258-71.

7. BRASIL. Ministério da Saúde. Secretaria de Vigilância em Saúde. Secretaria de Gestão Estratégica e Participativa. VIGITEL Brasil 2012: Vigilância de fatores de risco e proteção para doenças crônicas por inquérito telefônico. Brasília: Ministério da Saúde, 2013.

8. Haskell WL, Lee IM, Pate RR, Powell KE, Blair SN, Franklin BA, et al. Physical activity and public health: updated recommendation for adults from the American College of Sports Medicine and the American Heart Association. Med Sci Sports Exerc. 2007;39(8):1423-34.

9. World Health Organization. Global recommendations on physical activity for health. Geneva: World Health Organization, 2010.

10. Izrael D, Hoaglin DC, Battaglia MP. "A SAS Macro for Balancing a Weighted Sample." Proceedings of the Twenty-Fifth Annual SAS Users Group International Conference. SAS Institute Inc., 2000; 275: 1350-5.

11. IBGE. Instituto Brasileiro de Geografia e Estatística. Disponível em: http://downloads. ibge.gov.br/downloads_estatisticas.htm. Acessado em 17/11/2014.

12. BRASIL, Ministério da Saúde, Secretaria de Vigilância em Saúde. Saúde Brasil 2013: uma análise da situação de saúde e das doenças transmissíveis relacionadas à pobreza. Brasília: Ministério da Saúde, 2014.

13. Malta DC, Andrade SSA, Santos MAS, Rodrigues GBA, Mielke GI. Tendências dos indicadores de atividade física em adultos, conjunto de capitais do Brasil 20062013.2014; Rev Bras de Ativ Fís Saúde, 20(2):141-151.

14. Malta DC, Andrade SSA, Santos MAS , Campos MO, Rodrigues GBA, Nunes ML, et al. 2014; Rev Bras de Ativ Fís Saúde. (no prelo).

15. Silva ICM, Santin-Medeiros F, Bertapelli F, Ana Paula Coelho APS, Silva SG. Pesquisa em atividade física e saúde no Brasil: dimensão atual dos investimentos em projetos e bolsas de produtividade do CNPq. 2014; Rev Bras de Ativ Fís Saúde;19(3):325-32.

16. Garcia LMT, Böhm AW. Bacil EDA, Cruz, MF, Santo RCE. A inserção da subárea de Atividade Física e Saúde nos programas de pós-graduação em Educação Física no 
Brasil. 2014; Rev Bras de Ativ Fís Saúde;19(2):215-22.

17. Amorim TC, Knuth AG, Cruz DKA, Malta DC, Reis RS, Hallal PC. Descrição dos programas municipais de promoção da atividade física financiados pelo Ministério da Saúde. 2013; Rev Bras de Ativ Fís Saúde;18(1):63-74.

18. Mielke GI, Silva IC, Owen N, Hallal PC. Brazilian adults' sedentary behaviors by life domain: population-based study. PlosOne. 2014; 9(3): e91614.

19. Hallal PC, Cordeira KL, Knuth AG, Mielke GI, Victora CG. Ten-year trends in total physical activity practice in Brazilian adults: 2002-2012. 2014; J Phys Act Health. 2014;11(8):1525-30.

20. Malta DC, Neto OLM, Junir JBS. Presentation of the strategic action plan for coping with chronic diseases in Brazil from 2011 to 2022. 2011; Epidemiol Serv Saude; 20:425-438.

21. Brasil. Ministério da Saúde. Secretaria de Vigilância em Saúde. Secretaria de Atenção à Saúde. Política Nacional de Promoção da Saúde. Disponível em: http://bvsms.saude. gov.br/bvs/publicacoes/politica_nacional_promocao_saude_3ed.pdf. Acessado em $17 / 11 / 2014$.

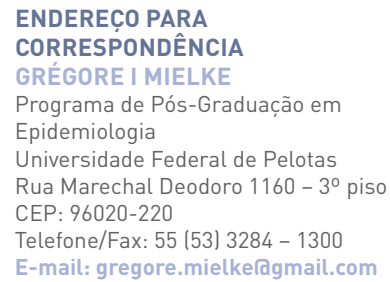

